\title{
Highly sensitive and specific novel biomarkers for the diagnosis of transitional bladder carcinoma
}

\author{
Prashant Kumar ${ }^{1, *}$, Sayantani Nandi ${ }^{1, *}$, Tuan Zea Tan ${ }^{2, *}$, Siok Ghee Ler ${ }^{3}$, Kee Seng \\ Chia $^{4}$, Wei-Yen Lim ${ }^{5}$, Zentia Bütow ${ }^{6}$, Dimitrios Vordos ${ }^{6}$, Alexandre De laTaille ${ }^{6}$, \\ Muthafar Al-Haddawi ${ }^{1}$, Manfred Raida7, Burkhard Beyer ${ }^{8}$, Estelle Ricci ${ }^{9}$, Marc \\ Colombel $^{9}$, Tsung Wen Chong ${ }^{10}$, Edmund Chiong ${ }^{11}$, Ross Soo ${ }^{12}$, Mi Kyoung Park ${ }^{13}$, \\ Hong Koo Ha ${ }^{14, * *}$, Jayantha Gunaratne ${ }^{3,4, * *}$ and Jean Paul Thiery ${ }^{1,2,7, * *}$ \\ ${ }^{1}$ Institute of Molecular and Cell Biology, Agency for Science, Technology and Research (A*STAR), Singapore \\ ${ }^{2}$ Cancer Science Institute, National University of Singapore, Singapore \\ ${ }^{3}$ Quantitative Proteomics Group, Institute of Molecular and Cell Biology, Agency for Science, Technology and Research, \\ Singapore \\ ${ }^{4}$ Department of Anatomy, Yong Loo Lin School of Medicine, National University of Singapore, Singapore \\ ${ }^{5}$ Saw Swee Hock School of Public Health, National University of Singapore, Singapore \\ ${ }^{6} \mathrm{CHU}$ Hopital Henri Mondor, Department of Urology, Créteil, France \\ ${ }^{7}$ Department of Biochemistry, Yong Loo Lin School of Medicine, National University of Singapore, Singapore \\ ${ }^{8}$ Department of Urology, Section for Translational Prostate Cancer Research, University Medical Center Hamburg-Eppendorf, \\ Hamburg, Germany \\ ${ }^{9}$ Service d'Urologie et Chirurgie de la Transplantation, Hôpital Edouard Herriot, Lyon, France \\ ${ }^{10}$ Department of Urology, Singapore General Hospital, Singapore \\ ${ }^{11}$ Department of Urology, University Surgical Cluster, National University Health System (NUHS), Singapore \\ 12 Haematology-Oncology Research Group, Department of Haematology-Oncology, National University Cancer Institute \\ (NCIS), National University Hospital, Singapore \\ ${ }^{13}$ Institute of Microelectronics (IME), A*STAR, Singapore \\ 14 Department of Urology, Pusan National University Hospital, Pusan National University School of Medicine, Busan, Korea \\ * These authors are co-first authors \\ ** These authors are co-senior authors \\ Correspondence to: Jean Paul Thiery, email: jpthiery@imcb.a-star.edu.sg
}

Keywords: transitional bladder carcinoma, urine biomarkers, receiving operating characteristics, combination model

Received: December 08, $2014 \quad$ Accepted: March 19, $2015 \quad$ Published: April 15, 2015

This is an open-access article distributed under the terms of the Creative Commons Attribution License, which permits unrestricted use, distribution, and reproduction in any medium, provided the original author and source are credited.

ABSTRACT

Transitional bladder carcinoma (BCa) is prevalent in developed countries, particularly among men. Given that these tumors frequently recur or progress, the early detection and subsequent monitoring of $\mathrm{BCa}$ at different stages is critical. Current BCa diagnostic biomarkers are not sufficiently sensitive for substituting or complementing invasive cystoscopy. Here, we sought to identify a robust set of urine biomarkers for BCa detection. Using a high-resolution, mass spectrometry-based, quantitative proteomics approach, we measured, compared and validated protein variations in $\mathbf{4 5 1}$ voided urine samples from healthy subjects, non-bladder cancer patients and patients with non-invasive and invasive $B C a$. We identified five robust biomarkers: Coronin-1A, Apolipoprotein A4, Semenogelin-2, Gamma synuclein and DJ-1/PARK7. In diagnosing Ta/T1 BCa, these biomarkers achieved an AUC of 0.92 and 0.98 , respectively, using ELISA and western blot data (sensitivity, 79.2\% and 93.9\%; specificity, $100 \%$ and $96.7 \%$, respectively). In diagnosing $\mathrm{T} 2 / \mathrm{T} 3 \mathrm{BCa}$, an AUC of 0.94 and 1.0 was attained (sensitivity, $86.4 \%$ and $100 \%$; specificity, $100 \%$ ) using the 


\section{same methods. Thus, our multiplex biomarker panel offers unprecedented accuracy for the diagnosis of $\mathrm{BCa}$ patients and provides the prospect for a non-invasive way to detect bladder cancer.}

\section{INTRODUCTION}

Transitional bladder carcinoma (BCa) is the fifthmost common cancer and results in significant morbidity and mortality. Cystoscopy and voided urine cytology are still the gold standards for the detection and follow-up of $\mathrm{BCa}[1]$ but they cannot detect certain lesions, such as small carcinoma in situ, and are often employed whenever patients present with other clinical signs. BCa exhibits significant tumor heterogeneity, reflected by multiple genetic alterations and complex somatic mutational profiles [2]. New biomarkers, based on DNA methylation profiling [3], point mutations [4] and microRNAs [5], have been established in addition to many proposed protein biomarkers.

Commercial tests that detect Nuclear Matrix Protein 22 (NMP22) [6] or Bladder Tumor Antigen (BTA) [7] are FDA-approved tests for $\mathrm{BCa}$ diagnosis, but these singlemarker assays lack the specificity of voided urine cytology [8]. In addition, two protein signatures comprising ten [9] or eight [10] biomarkers were developed more recently for the detection of recurrent $\mathrm{BCa}$. However, none of these diagnostic marker assays offer sufficient sensitivity and specificity to be routinely used in the clinic. It is thus desirable to identify an optimal number of highly specific biomarkers that, in combination, can improve the robustness of the currently available diagnostic methodologies.

Advanced mass spectrometry (MS)-based quantitative proteomics has emerged as a powerful technology that delivers accurate and unbiased information about the quantitative behavior of a wide variety of proteins in complex biological samples. Thus, the aim of our study was to establish a multi-analyte assay for non-muscle invasive (Ta/T1) and muscle invasive (T2/T3) BCa detection in urine using MS technology in combination with multiplex peptide stable isotope labeling (reductive dimethyl labeling strategy) — a novel approach for $\mathrm{BCa}$ biomarker discovery - to capture differentially secreted proteins in $\mathrm{BCa}$ patients. We identified five highly sensitive and specific biomarkers, which were validated in urine samples from a large cohort of patients with $\mathrm{BCa}$, benign urological conditions, chronic diseases or other cancer types.

\section{RESULTS}

\section{Overview of candidate biomarker identification for $\mathrm{BCa}$ detection using quantitative MS}

To identify potential biomarkers for the diagnosis of $\mathrm{Ta} / \mathrm{T} 1$ and $\mathrm{T} 2 / \mathrm{T} 3 \mathrm{BCa}$, we performed MS analysis using pooled urine samples of equal volumes from four subjects. In order to minimize experimental errors, we included biological (different pools) and technical replicates (different MS runs for the same samples). We combined the entire dataset to obtain the mean fold change in proteins present in $\mathrm{BCa}$ urine samples as compared with healthy urine samples. Proteins with at least a twofold and two ratio count difference were considered as over-secreted proteins, and these were then scrutinized through extensive online database literature searching. These proteins were shortlisted to 17 potential hits based on their novelty, previously published cancer association, and subcellular localization.

\section{Identification of five candidate biomarkers for $B C a$ in voided urine samples}

Shortlisted, potential biomarkers were next considered for initial validation by RT-PCR with ten samples for each of the three groups: control (healthy subjects), Ta/T1, and T2/T3 BCa. Based on the results of the mRNA expression analysis (data not shown), we found a higher expression (fold change $>2$ ) of five candidate biomarkers-Coronin-1A, Apolipoprotein A4, Semenogelin-2, Gamma Synuclein and DJ-1-in Ta/T1 and T2/T3 BCa samples as compared with samples from healthy subjects (Supplementary Figure 1). The shortlisted potential biomarkers were also validated using western blot analysis of healthy subjects and $\mathrm{BCa}$ patient urine samples before determining the five biomarkers.

\section{Validation of five candidate biomarkers for BCa in a larger cohort}

Urine samples from 66 healthy subjects, $110 \mathrm{Ta} / \mathrm{T} 1$ $\mathrm{BCa}$ patients and $63 \mathrm{~T} 2 / \mathrm{T} 3 \mathrm{BCa}$ patients were analyzed using western blotting. All $\mathrm{BCa}$ urine samples exhibited an elevated level in at least three out of the five biomarkers (Figure 1). ROC curve, established using band intensity values, showed an AUC of 0.98 and 1.0 for healthy versus $\mathrm{Ta} / \mathrm{T} 1$ and healthy versus $\mathrm{T} 2 / \mathrm{T} 3$, respectively, using the 

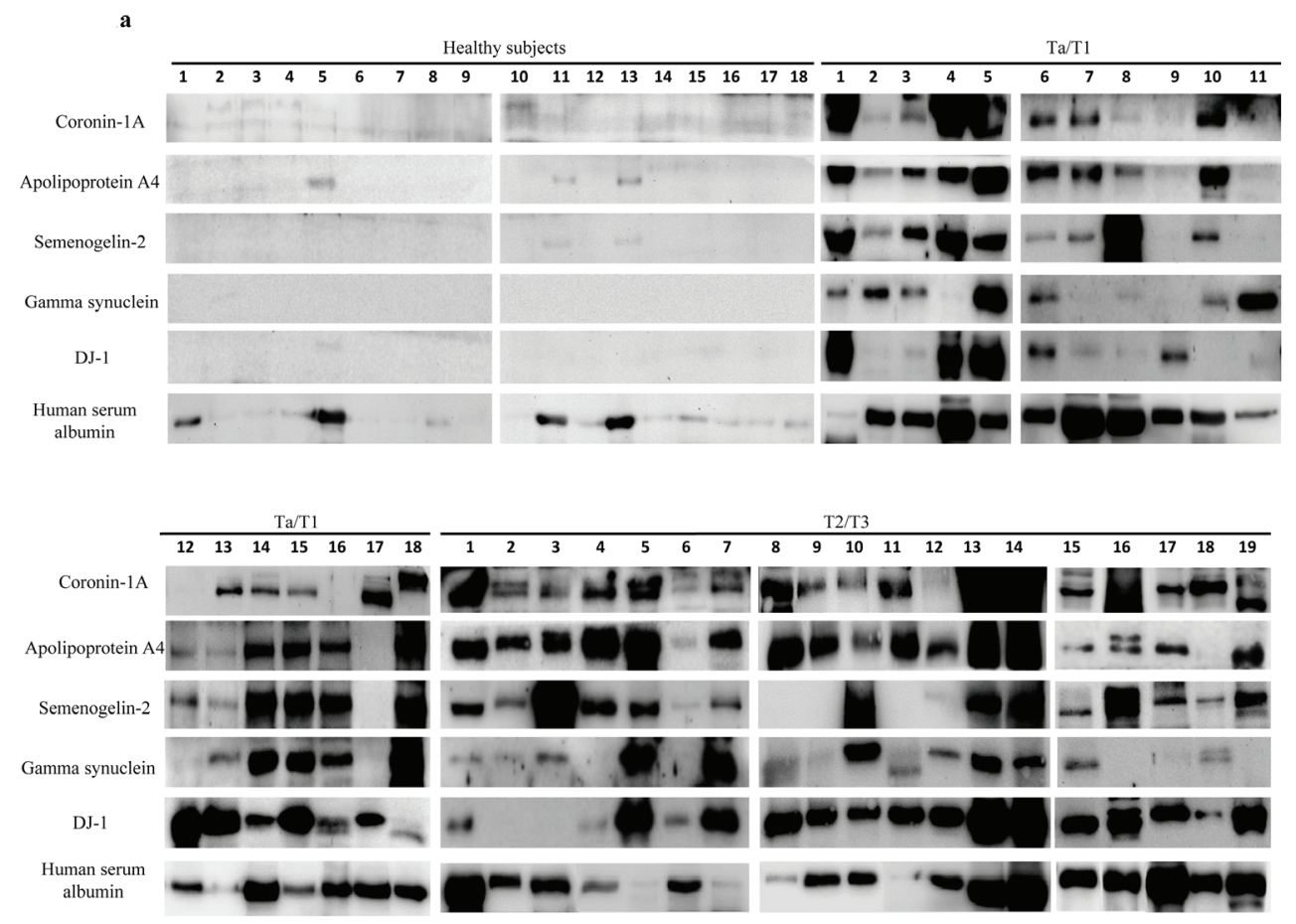

b
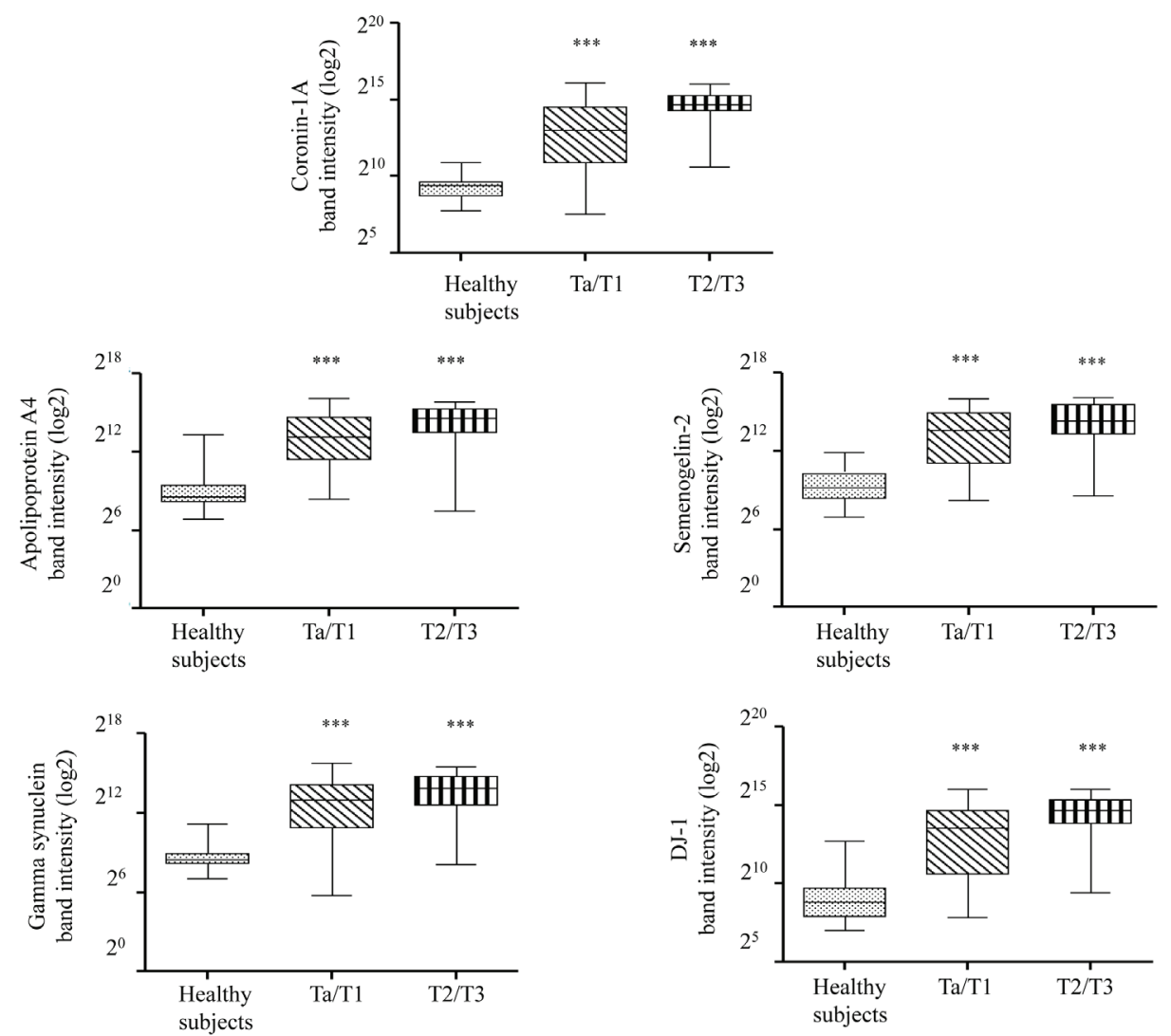

Figure 1: Western blot analysis of urine samples from healthy subjects and bladder carcinoma (BCa) patients. a. Protein $(100 \mu \mathrm{g})$ from each voided urine sample of healthy subjects, non-muscle invasive (NMI; Ta/T1) and muscle invasive (MI; T2/ T3) BCa patients was subjected to western blotting. Human serum albumin (HSA) protein was also examined in each sample to assess for a correlation between biomarker expression and hematuria. b. Box-and-Whisker plot of log2 band intensities of the blots plotted for the five biomarkers from all voided urine samples obtained from healthy subjects $(n=66)$, Ta/T1 $(n=110)$ and T2/T3 $(n=63) \mathrm{BCa}$ patients. The box represents the lower quartile, median, and higher quartile values; the whiskers show the minimum and maximum values. MannWhitney test was used to compute significance. $* * * p<0.001$ (as compared with healthy samples). 
Table 1A: Accuracy of the combination model in diagnosing Ta/T1 bladder cancer.

\begin{tabular}{|c|c|c|c|c|c|c|c|}
\hline \multicolumn{2}{|r|}{ Biomarkers } & AUC & Sensitivity \% & $\begin{array}{c}\text { Specificity } \\
\%\end{array}$ & PPV \% & $\begin{array}{r}\text { NPV } \\
\%\end{array}$ & $\begin{array}{l}\text { Overall } \\
\text { accuracy } \%\end{array}$ \\
\hline ELISA & $\begin{array}{c}\text { All five biomarkers in } \\
\text { combination }\end{array}$ & 0.92 & 79.2 & 100 & 100 & 66.7 & 85.3 \\
\hline \multirow{3}{*}{ Western blot } & $\begin{array}{c}\text { All five biomarkers in } \\
\text { combination }\end{array}$ & 0.98 & 93.9 & 96.7 & 98.4 & 87.9 & 94.8 \\
\hline & $\begin{array}{l}\text { Three biomarkers } \\
\text { (Coronin-1A, } \\
\text { Apolipoprotein A4, DJ- } \\
\text { 1) in combination }\end{array}$ & 0.97 & 92.4 & 93.3 & 96.8 & 84.8 & 92.7 \\
\hline & $\begin{array}{l}\text { Four biomarkers } \\
\text { (Coronin-1A, } \\
\text { Apolipoprotein A4, } \\
\text { Semanogelin-2, DJ-1) in } \\
\text { combination }\end{array}$ & 0.98 & 90.9 & 96.7 & 98.4 & 82.9 & 92.7 \\
\hline
\end{tabular}

Abbreviations: AUC, area under the curve; PPV, positive predictive value; NPV, negative predictive value.

Table 1B: Accuracy of the combination model in diagnosing T2/T3 bladder cancer.

\begin{tabular}{|c|c|c|c|c|c|c|c|}
\hline \multicolumn{2}{|r|}{ Biomarkers } & $\mathbf{A U C}$ & Sensitivity \% & $\begin{array}{c}\text { Specificity } \\
\%\end{array}$ & PPV \% & $\begin{array}{r}\text { NPV } \\
\%\end{array}$ & $\begin{array}{l}\text { Overall } \\
\text { accuracy } \%\end{array}$ \\
\hline ELISA & $\begin{array}{c}\text { All five biomarkers in } \\
\text { combination }\end{array}$ & 0.94 & 86.4 & 100 & 100 & 76.9 & 90.6 \\
\hline \multirow{3}{*}{ Western blot } & $\begin{array}{c}\text { All five biomarkers in } \\
\text { combination }\end{array}$ & 1.0 & 100 & 100 & 100 & 100 & 100 \\
\hline & $\begin{array}{l}\text { Three biomarkers (Coronin- } \\
\text { 1A, Apolipoprotein A4, DJ- } \\
\text { 1) in combination }\end{array}$ & 1.0 & 100 & 100 & 100 & 100 & 100 \\
\hline & $\begin{array}{l}\text { Four biomarkers (Coronin- } \\
\text { 1A, Apolipoprotein A4, } \\
\text { Semanogelin-2, DJ-1) in } \\
\text { combination }\end{array}$ & 1.0 & 100 & 100 & 100 & 100 & 100 \\
\hline
\end{tabular}

Abbreviations: AUC, area under the curve; PPV, positive predictive value; NPV, negative predictive value.

Table 2: Pearson correlation analysis of blood plasma and white blood cells with biomarkers.

\begin{tabular}{|c|c|c|c|}
\hline \multirow{2}{*}{ Biomarkers } & Healthy Subjects & $\begin{array}{c}\text { Ta/T1 BCa Samples } \\
\text { (non-muscle invasive) }\end{array}$ & $\begin{array}{c}\text { T2/T3 BCa Samples } \\
\text { (muscle invasive) }\end{array}$ \\
\cline { 2 - 4 } & Pearson $\boldsymbol{r}$ & Pearson $\boldsymbol{r}$ & Pearson $\boldsymbol{r}$ \\
\hline Coronin-1A & +0.1874 & +0.3198 & +0.4320 \\
\hline Apolipoprotein A4 & +0.4570 & -0.1138 & +0.1753 \\
\hline Semenogelin-2 & +0.3112 & +0.1645 & +0.2603 \\
\hline Gamma synuclein & +0.3986 & -0.035 & -0.1208 \\
\hline DJ-1 & +0.3198 & +0.0092 & +0.1300 \\
\hline
\end{tabular}

Pearson correlation coefficient $(r)$ for the biomarkers in urine and blood plasma samples from healthy subjects, Ta/T1 BCa (non-muscle invasive), and T2/T3 BCa (muscle invasive) patients. Abbreviations: BCa, bladder carcinoma. 
five biomarkers in combination (Figure 3; Table 1A and 1B). The data from western blot analyses (Figure 1a and $1 \mathrm{~b}$ ) showed perfect concordance with the RT-PCR data (Supplementary Figure 1), indicating that the five biomarkers were significantly enriched in urine samples of BCa patients as compared with that of healthy subjects. Representative MS spectra (Supplementary Figure 2) of the five potential markers were also in good agreement with RT-PCR and western blotting data.

\section{Hematuria had no effect on urinary biomarker levels}

Hematuria occurs in numerous patients with urinary tract disease. We wanted to ascertain whether hematuria could affect a BCa diagnosis using our selected biomarkers, as these proteins can also be found in blood; albeit, at low levels. Supplementary Table 5, based on a database search, provides the prevalence and, in some cases, the large concentration range of four of our five biomarkers in plasma. We tested for the expression of these five biomarkers in plasma and in white blood cells from four blood samples using western blot analysis. We found the expression of all five biomarkers in plasma but only Coronin-1A in white blood cell extracts.

To compute the correlation of these biomarkers within plasma, we quantified the western blot intensity of the biomarkers in blood and urine samples and normalized them to human serum albumin (HSA) values in each respective sample. The Box-and-Whisker plot shows that all five biomarkers were significantly expressed in $\mathrm{Ta} /$ $\mathrm{T} 1$ and T2/T3 urine samples relative to that in samples from healthy subjects or in the blood plasma from four individual samples (Figure 2). Moreover, only Coronin1A showed marginal correlation with HSA levels using a
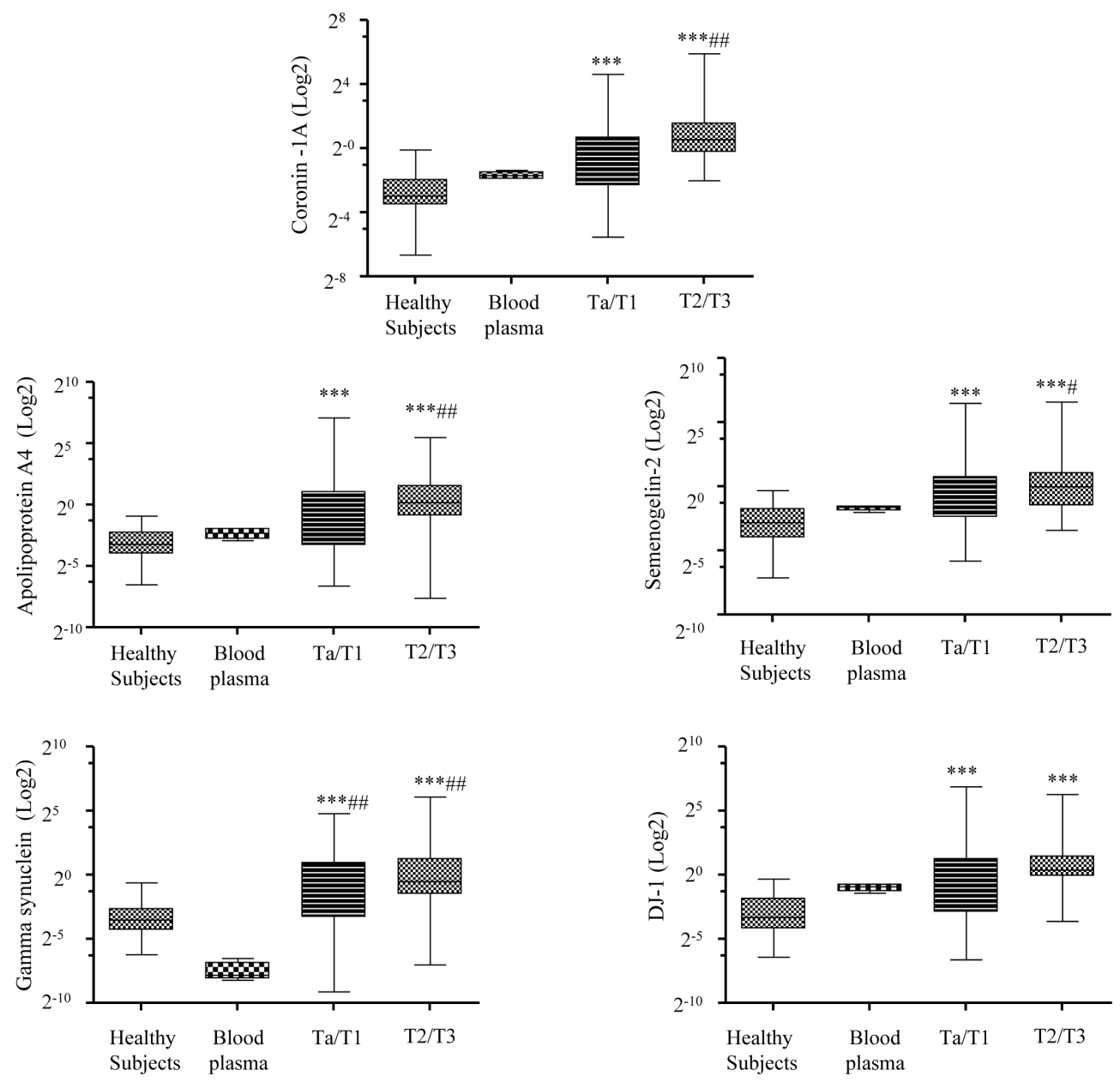

Figure 2: Correlation of biomarkers and hematuria in urine samples. Box-and-Whisker plot of the five biomarkers (Coronin1A, Apolipoprotein A4, Semenogelin-2, Gamma synuclein and DJ-1) with respect to albumin in voided urine samples from healthy subjects $(\boldsymbol{n}=30)$, blood plasma $(\boldsymbol{n}=4), \mathrm{Ta} / \mathrm{T} 1$ bladder carcinoma $(\mathrm{BCa})(\boldsymbol{n}=66)$, and T2/T3 BCa $(\boldsymbol{n}=28)$. The box represents the lower quartile, median, and higher quartile values. The whiskers show the minimum and maximum values. Mann-Whitney test was used to compute significance. ${ }^{*} p<0.05,{ }^{* *} p<0.01,{ }^{* * *} p<0.001$ as compared with healthy samples; ${ }^{*} p<0.05,{ }^{\#} p<0.01,{ }^{\# \#} p<0.001$ as compared with blood plasma samples. 
Pearson correlation test (Table 2). From these results, we conclude that our biomarkers were not expressed in urine as a result of hematuria.

We next sought to correlate Coronin-1A expression with white blood cell count, making the assumption that HSA originated only from blood rather than from defective kidney filtration. We found at least a 289 -fold increase in the actual band intensity of Coronin-1A in urine as compared to what could have theoretically originated from white blood cells (see Appendix A). This analysis thus allowed us to exclude any significant contribution of white blood cells to Coronin-1A content.

\section{Minimal biomarker prevalence in urine samples from non-bladder cancer patients}

To further evaluate the specificity of our biomarkers in $\mathrm{BCa}$, we performed western blotting analysis of 91 urine samples from non-bladder cancer patients and 121 urine samples from patients suffering from diverse chronic

a.

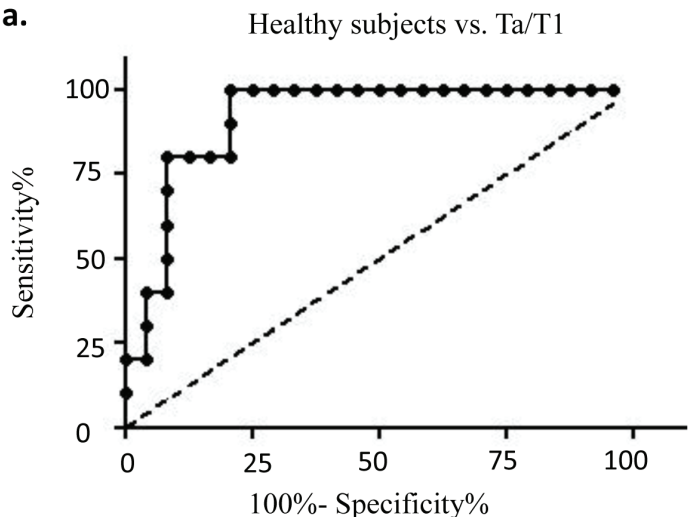

b. Healthy subjects vs. Ta/T1

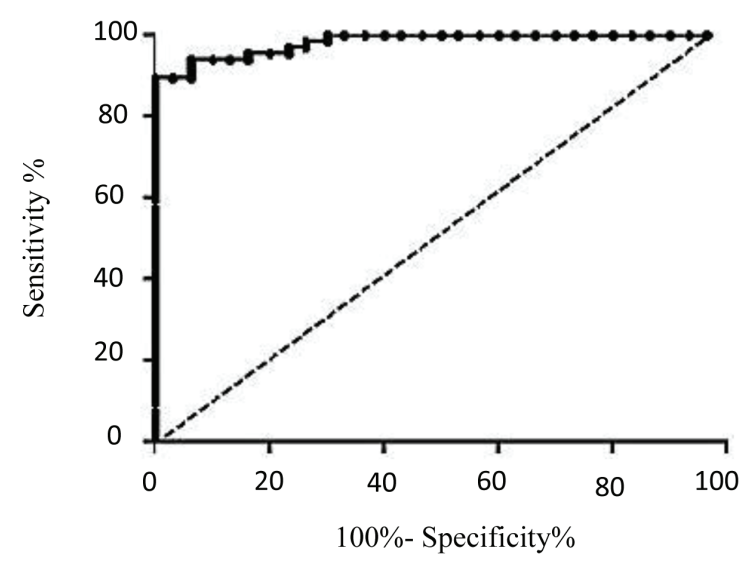

ailments (Supplementary Table 1). The vast majority of samples were completely negative for these five biomarkers; albeit, in approximately $5 \%$ of the samples, we noticed variable expression of only one or, rarely, two biomarkers (Supplementary Figures 3 and 4).

\section{Validation of the high sensitivity and specificity of our diagnostic model}

Finally, we developed a diagnostic model using Lasso regression to evaluate the clinical utility of different combinations of all five urine biomarkers. The diagnostic performances of each of the five biomarkers were computed separately and in various combinations using ELISA and western blotting data (Table 1A and 1B). Sensitivity and specificity, along with the cut-off values, for each biomarker are listed in Supplementary Tables 6A and $6 \mathrm{~B}$. Using ELISA expression data, we found that a diagnostic model that combined all five biomarkers was the most accurate, achieving an AUC of 0.92 and an
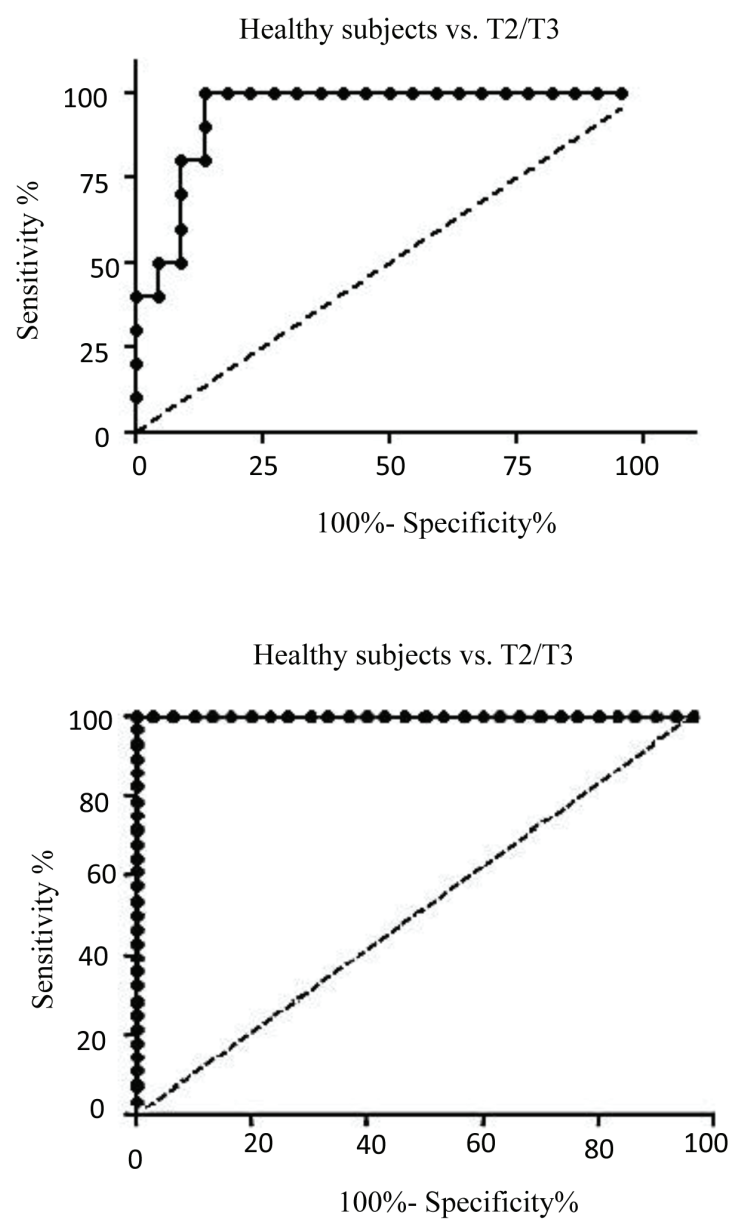

Figure 3: Receiver operating characteristic (ROC) curves designed to evaluate the accuracy of the multiplex biomarker model for the diagnosis of bladder carcinoma (BCa). This model assesses the efficacy of the biomarkers to distinguish both nonmuscle invasive (NMI; Ta/T1) and muscle invasive (MI; T2/T3) BCa from healthy subjects based on data obtained from a. ELISA analysis and $\mathbf{b}$. western blot analysis of voided urine samples. 
Appendix A: Calculations to demonstrate that the major contributor of Coronin-1A in BCa patient urine is not from lysed WBCs but from the bladder tumor.

\begin{tabular}{|c|c|}
\hline Albumin determination & \\
\hline Concentration of albumin in blood* & $40 \mu \mathrm{g} / \mu \mathrm{l}$ \\
\hline Concentration range of albumin in $\mathrm{BCa}$ patient urine samples ${ }^{\&}$ & $0.012-2.56 \mu \mathrm{g} / \mu \mathrm{l}$ \\
\hline Mean albumin concentration in BCa patient urine samples & $1.18 \mu \mathrm{g} / \mu \mathrm{l}$ \\
\hline Approximate volume of blood in urine ${ }^{\#}$ & $(1 / 40) \times 1.18=0.0295 \mu 1$ \\
\hline \multicolumn{2}{|l|}{ White Blood Cells (WBC) determination } \\
\hline No. of $\mathrm{WBC} / \mu \mathrm{l}$ of blood* & $\sim 5000 \mathrm{WBCs}$ \\
\hline Hence, $\sim 0.0295 \mu 1$ of blood in urine contains & $(5000 \times 0.0295)=147.5 \mathrm{WBCs}$ \\
\hline \multicolumn{2}{|l|}{ Coronin-1A calculation } \\
\hline Weight of a single cell* & $3.5 \times 10^{-3} \mu \mathrm{g}$ \\
\hline Amount of protein/cell ${ }^{\S} *$ & $0.0007 \mu \mathrm{g}$ \\
\hline $40 \mu \mathrm{g}$ of lysed WBC from buffy coat would be derived from & $(1 / 0.0007) \times 40 \approx 57,000 \mathrm{WBCs}^{*}$ \\
\hline Coronin-1A blot intensity of $57,000 \mathrm{WBC}$ lysate $£$ & 34,403 \\
\hline Hence, 147.5 WBCs if lysed in urine would produce intensity of & $(34,403 / 57000) \times 147.5=89.025$ \\
\hline Actual mean Coronin-1A intensity from BCa urine sample blot & $25,788.58^{4}$ \\
\hline Fold-change in intensity for Coronin-1A & $(25,788.58 / 89.025)=289.68$ \\
\hline
\end{tabular}

${ }^{*}$ Reported in the literature

\&Deduced from our experimental analysis; further calculations were done using the higher limit of the range.

\#Assumption: Albumin source is solely blood §Protein weight is $20 \%$ of cells weight

${ }^{\ddagger}$ Rounded down for calculations. Actual calculation is $57,142.86 \mathrm{WBCs}$

${ }^{\mathfrak{f}}$ Result obtained from western blotting experiments conducted in our lab

"Averaged value

overall accuracy of $85.3 \%$ (sensitivity, $79.2 \%$; specificity, $100 \%$ ) in differentiating $\mathrm{Ta} / \mathrm{T} 1 \mathrm{BCa}$ patients from healthy subjects. Similarly, for diagnosing $\mathrm{T} 2 / \mathrm{T} 3 \mathrm{BCa}$, the diagnostic model achieved an AUC of 0.94 and an overall accuracy of $90.6 \%$ (sensitivity, $86.4 \%$; specificity, $100 \%$ ); as shown in Figure 3 and Tables 1A and 1B. Likewise, using western blotting data, we obtained an AUC of 0.98 and an overall accuracy of $94.8 \%$ (sensitivity, $93.9 \%$; specificity, 96.7\%) for a $\mathrm{Ta} / \mathrm{T} 1 \mathrm{BCa}$ diagnosis, and, surprisingly, an AUC of 1.0 and an overall accuracy of $100 \%$ for a T2/T3 BCa diagnosis (Figure 3 and Tables $1 \mathrm{~A}$ and 1B). Thus, our diagnostic model combining all five biomarkers is highly sensitive and specific, outperforming conventional cytology or any existing biomarkers reported to date for a $\mathrm{Ta} / \mathrm{T} 1 \mathrm{BCa}$ diagnosis [13]. Our biomarker panel proved to be superior to the cytology data we obtained from clinics for the healthy subject and $\mathrm{BCa}$ patients (Supplementary Table 1).
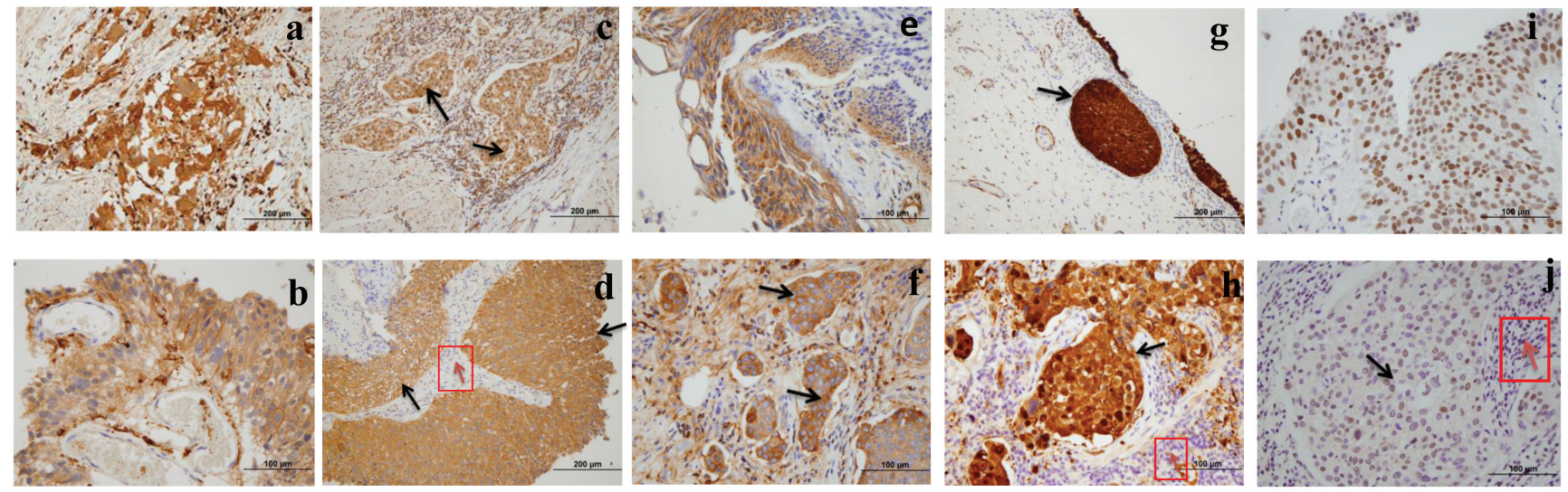

Figure 4: High expression of five biomarkers in bladder carcinoma (BCa) tissue. a. Clusters of invasive neoplastic cells staining positively for Coronin-1A. b. Coronin-1A positively stained carcinoma in situ (CIS). c. DJ-1 positively stained clusters of invasive carcinoma (black arrow). d. DJ-1 positively stained bladder carcinoma (black arrow) and negatively stained inflammatory cells (red arrow). e. APOA4 positively stained CIS. f. APOA4 positively stained clusters of invasive neoplasm (black arrow). g. Gamma-synuclein positively stained CIS (black arrow). h. Gamma-synuclein positively stained invasive neoplasm (black arrow) and negatively stained inflammatory cells (red arrow). i. Semenogelin-2 positively stained CIS. j. Semenogelin-2 positively stained invasive neoplastic cells (black arrow) and negatively stained inflammatory cells (red arrow). 


\section{DISCUSSION}

Advanced, MS-based, quantitative proteomics allowed us to identify a panel of novel biomarkers with high precision for $\mathrm{BCa}$ diagnosis. The five potential candidates were shortlisted from MS data through extensive published literature filtering followed by RTPCR, western blotting analyses and ELISA validation. The results showed elevated urinary concentrations of these five biomarkers in patients with $\mathrm{BCa}$, with equally enriched expression found in immunohistochemically stained BCa tissues (Figure 4). Each of these five proteins has an important biological function, as shown by the relevant literature in Supplementary Table 7. Coronin$1 \mathrm{~A}$ is a crucial component of the actin cytoskeleton and promotes cellular processes including endocytosis and cell motility. It is expressed in brain, thymus, spleen, bone marrow and lymph nodes, and selective cytoplasmic expression has been reported in leukocytes and microglia.

Apolipoprotein A4 (APOA4), a critical mediator of lipid metabolism, is also proposed to be a mediator of reverse cholesterol transport (removes cholesterol from peripheral cells and directs it to the liver for metabolism). In addition, it is regarded as an antioxidant, a mediator of gut inflammation, and has been linked to $\beta$-amyloid clearance in the brain. Serum APOA4 is a reported marker of pancreatic [12] and ovarian [14] cancers, and it is differentially expressed in the sera of gastric cancer patients [15]. APOA4 is elevated in patients with chronic kidney disease [16], and APOA1 in diabetic rat bladders [17].

The third biomarker, Gamma synuclein belongs to a family of small, soluble proteins consisting of $\alpha$-, $\beta$ - and $\gamma$-synuclein. Synucleins have been implicated in neurodegenerative diseases, but the function of $\gamma$-synuclein is not completely understood. $\gamma$-Synuclein overexpression is known to cause an increase in proliferation, invasiveness and metastasis [18], and $\gamma$-synuclein, along with calreticulin and catechol-o-methyltransferase, is reported to be a tumor marker in $\mathrm{BCa}$ [19].

Semenogelin-2, the fourth marker, is a major coagulating protein in human semen. Although semenogelin expression is normally restricted to males, two small cell lung carcinoma cell lines from female patients have been reported positive for semenogelin II expression [20]. The role of semenogelins in human malignancy is poorly understood; however, various studies have reported the expression of semenogelins in lung carcinomas, melanoma and leukemias. Semenogelin II has been shown to have prognostic significance in the prediction of cancer progression after radical prostatectomy [21].

Finally, DJ-1 is a conserved protein, coded by the gene Parkinson disease 7 (PARK7). DJ-1 was originally identified as an oncogene that can transform mouse NIH3T3 cells in cooperation with H-RAS [22]. DJ-1 protein affects cell survival by modulating cellular signaling cascades, such as the PTEN-phosphatidylinositol 3-kinase-Akt pathway [23], and can alter the activity of p53 [24]. The elevation of serum DJ-1 levels in patients with pancreatic cancer has led to the identification of DJ-1 as a potential diagnostic marker [25]. DJ-1 expression is correlated with the pathological stages of invasive urothelial carcinoma of the urinary bladder [26].

The diagnostic performance of these five biomarkers indicated a necessity to combine all five for an accurate diagnosis. FDA-approved Complement factor $\mathrm{H}$, which has been reported for the diagnosis of patients with benign renal diseases and urinary tract infections as well as other cancer types, provides only $60 \%$ specificity [27]. Our five urine biomarkers were able to differentiate patients with $\mathrm{BCa}$ from those with benign conditions (such as inflammation of the bladder, benign prostatic hyperplasia or nephrolithiasis possibly associated with hematuria) with $100 \%$ specificity, and were rarely detected in the urine of patients affected by chronic diseases or other cancer types.

The FDA-approved biomarker NMP22 is reported to be affected by hematuria in urine [28]. Hence, we took this issue into consideration during our validation tests. Normal HSA levels in urine vary considerably and can be detected in samples from otherwise healthy subjects. Notably, urine from patients with chronic diseases, such as diabetes, has a much higher level of HSA than that from many of our BCa patients. Therefore, HSA does not always reflect blood contamination. In addition, plasma or white blood cell contamination cannot account for the levels of these biomarkers detected following low-speed centrifugation of urine.

A follow-up study during the course of treatment or with post-treatment patients will be needed to test whether the combination of these five biomarkers can predict patient outcome or detect $\mathrm{BCa}$ recurrence. This is particularly critical in patients with superficial $\mathrm{BCa}$ who are treated with trans-urethral resection or who undergo Bacillus Calmette-Guerin (BCG) treatment.

\section{CONCLUSIONS}

We established a robust, urinary protein biomarker panel for $\mathrm{BCa}$ diagnosis. This panel consists of five biomarkers with significantly higher sensitivity and specificity than that of the currently available, noninvasive diagnostic assays for the detection of $\mathrm{BCa}$. This panel has the potential to be used to not only monitor and follow-up patients with $\mathrm{BCa}$, but also as a tool for screening asymptomatic subjects who are at a high risk of developing BCa. This study is likely to be immensely beneficial to patients as a companion diagnostic in the standard of care or as a potential novel therapeutic.

Non-standard Abbreviations: $\mathrm{BCa}$, bladder cancer; ROC, receiver operating characteristic; MS, mass spectrometry; HSA, human serum albumin; CIS, 
carcinoma in situ.

\section{MATERIALS AND METHODS}

\section{Urine samples}

Voided urine samples and associated clinical information (Supplementary Table 1) were collected following Institutional Review Board approval and informed consent. Samples were obtained from Department of Urology, National University Hospital (IRB \#02 235); National University Health System Tissue Repository (IRB B-14-015E); Department of Urology, Singapore General Hospital (IRB 2012-525); Department of Urology, CHU Henri Mondor Creteil, France (IRB 07284); Department of Urology, Hospital Lyon CHU Edouard Herriot Hospital (IRB 2008-073); Department of Urology, Medical School Hamburg, Germany (IRB PV3652) and Department of Urology, Pusan National University Hospital, Pusan, South Korea (IRB PNUH-E-2014118).

For the initial screening, $20 \mathrm{ml}$ of voided urine was collected from healthy subjects and $\mathrm{BCa}$ patients in preservation tubes (Norgen Biotek Corporation, Canada) and stored at $-80^{\circ} \mathrm{C}$. The voided urine was cleancatch, midstream urine, and the samples did not contain squamous cells or microbes. The samples were from three separate groups: the control group, corresponding to healthy subjects with no previous history of urothelial cell carcinoma, gross haematuria, active urinary tract infection or urolithiasis; a second group, formed by $\mathrm{Ta} /$ $\mathrm{T} 1 \mathrm{BCa}$ patients, characterized as having non-muscle invasive (NMI) $\mathrm{BCa}$; and a third group, comprising $\mathrm{T} 2 /$ T3 BCa patients, characterized as having muscle invasive (MI) BCa. Each urine aliquot was assigned a unique identifier before laboratory processing. Urine samples were excluded if they had significant blood contamination, as determined by visual inspection and based on the Dipstick urine test (Combur9, Roche Diagnostics, Basel, Switzerland) [results tabulated in Supplementary Table 2]. Samples were stored at $-80^{\circ} \mathrm{C}$ until further processing. For validation screens, an additional $5 \mathrm{ml}$ of voided urine was collected from patients affected by chronic diseases, $\mathrm{BCa}$, and other cancer types. Control urine samples were obtained from healthy, community-dwelling participants of the Singapore Consortium of Cohort Studies.

\section{Mass spectrometry analysis}

Concentrated urine samples were subjected to oncolumn stable isotope dimethyl labeling followed by nLC-MS analysis. Urine proteins were concentrated using a 3-kDa centrifugal filter, according to the manufacturer's instructions (Millipore, Carrigtwohill, Ireland). Four samples from each cohort, i.e., healthy, Ta/T1 and $\mathrm{T} 2 /$ $\mathrm{T} 3 \mathrm{BCa}$, were pooled and centrifuged at $8000 \times g$ for 30-60 $\mathrm{min}$ at $4^{\circ} \mathrm{C}$. Acetone-precipitated samples were reconstituted in $120 \mu \mathrm{l}$ of $8 \mathrm{M}$ urea in $100 \mathrm{mM}$ ammonium bicarbonate. Samples were reduced by the addition of 1 $\mathrm{M}$ dithiothreitol to a final concentration of $5 \mathrm{mM}$ in each sample, and then incubated at room temperature for 30 min. Alkylation was performed by the addition of $0.5 \mathrm{M}$ iodoacetamide to a final concentration of $10 \mathrm{mM}$, and the samples were incubated in the dark for $30 \mathrm{~min}$. All samples were then diluted from the initial $8 \mathrm{M}$ urea to 6 $\mathrm{M}$ urea using $100 \mathrm{mM}$ ammonium bicarbonate and then incubated with Lys-C (enzyme-to-protein ratio of 1:100) at $37^{\circ} \mathrm{C}$ overnight. Digestion mixtures were adjusted to a final concentration of $1 \mathrm{M}$ urea by the addition of 50 $\mathrm{mM}$ ammonium bicarbonate followed by incubation with trypsin (enzyme-to-protein ratio of $1: 50$ ) at $37^{\circ} \mathrm{C}$ for $4 \mathrm{~h}$.

The tryptic peptide mixtures were subjected to oncolumn stable isotope dimethyl labelling, as described elsewhere [11], using a triple-labelling approach. Differentially labelled urine samples were mixed and fractionated using isoelectric focusing electrophoresis on an Agilent 3100 OFFGEL Fractionator (12 fractions). The samples were cleaned up using self-packed $\mathrm{C} 18$ stage tips and subjected to mass spectrometry analysis, also as previously described [11].

MS data were analyzed by MaxQuant version 1.3.0.5 using the 2013-07_uniprot human FASTA database (88354 entries) [12]. Maximum false discovery rates were set to 0.01 for both protein and peptide. Proteins were considered identified when supported by at least one unique peptide with a minimum length of 7 amino acids.

\section{Quantitative real-time polymerase chain reaction}

Total RNA was isolated from sloughed cells within the $5 \mathrm{ml}$ urine samples using the ZR Urine RNA Isolation Kit (Zymo Research, Irvine, CA). Total RNA (500 ng) was reverse transcribed using SuperScript ${ }^{\circledR}$ III FirstStrand Synthesis System (Invitrogen, Carlsbad, CA). RTPCR pre-amplification reactions were carried out using EXPRESS SYBR ${ }^{\circledR}$ GreenER ${ }^{\text {TM }}$ qPCR Supermix with Premixed ROX (Invitrogen). The primer sequences are listed in Supplementary Table 3.

\section{Western blot analysis}

Western blotting was carried out on urine samples after they were centrifuged to eliminate cells. Protein concentrations were estimated using a BCA Protein Assay Kit (Pierce, Rockford, IL). Proteins $(100 \mu \mathrm{g})$ from individual samples were resolved on sodium dodecyl sulphate (SDS)-polyacrylamide gel electrophoresis (PAGE) and transferred to polyvinylidene difluoride (PVDF) membranes (Bio-Rad Laboratories, Hercules, 
CA). Membranes were blocked with 5\% bovine serum albumin (BSA) in Tris-buffered saline containing $0.1 \%$ Tween-20 (TBST) for $1 \mathrm{~h}$ at room temperature followed by probing with primary antibodies listed in Supplementary Table 4.

\section{Protein quantification by ELISA}

Urinary protein concentration for each biomarker was determined using respective ELISA kits (USCN Lifescience Inc., Wuhan, Hubei) according to the manufacturer's instructions (in triplicates). The kits are listed in Supplementary Table 4. The biomarker quantity in the urine was normalized by loading an equal concentration of total protein into each well of the ELISA plate.

\section{Histopathology}

BCa biopsy samples were fixed in 10\% neutralbuffered formalin for $48 \mathrm{~h}$, transferred to $70 \%$ ethanol, and then embedded in paraffin. Tissue sections $(4 \mu \mathrm{m})$ were stained with hematoxylin and eosin for histopathological examination. Heat-induced epitope retrieval was performed using Bond ${ }^{\mathrm{TM}}$ Epitope Retrieval Solution 2 ( $\mathrm{pH}$ 9.0) for $40 \mathrm{~min}$ at $100^{\circ} \mathrm{C}$. Immunohistochemical staining was performed using the Leica Bond ${ }^{\mathrm{TM}}$ Autostainer, which uses their proprietary Bond ${ }^{\mathrm{TM}}$ Detection Refine Kit (Leica, Solms, Germany). All immunofluorescence labelling was performed using antibody dilutions listed in Supplementary Table 4.

\section{Statistical analysis}

Significance evaluation for mean differences and correlations were computed by an unpaired $t$-test and Pearson correlation analysis, respectively, using Matlab ${ }^{\circledR}$ R2012a (MathWorks, Natick, MA). Band intensity from western blots was quantified using ImageJ (National Institutes of Health, Bethesda, MD). A diagnostic model combining the five biomarkers was developed using the Lasso regression model from Matlab ${ }^{\circledR}$ R2012a statistical toolbox version 8.0 (MathWorks, Natick, MA). Calibration curves were prepared using purified proteins provided with the ELISA kits. Receiver Operating Characteristic (ROC) curves and AUC were computed using Graphpad Prism $^{\circledR}$ version 5.0 (GraphPad Software, La Jolla, CA). Thresholds for each biomarker were selected based on Youden's Index. Sensitivity, specificity, positive predictive value (PPV), negative predictive value (NPV), and overall accuracy were computed using the selected threshold for diagnosis. A $P$-value $<0.05$ (two-sided) was regarded as significant.

\section{ACKNOWLEDGEMENTS}

We are grateful to all participating patients, clinicians and their staff for their kind assistance in the collection of urine and for the provision of tumor tissue blocks and patient data. We also appreciate the assistance in technical support and facility usage from the Histopathology Facility/Advanced Molecular Pathology Lab (AMPL), IMCB; Dr. Farazeela Mohd Ibrahim for histological processing and staining; and Dr. R. A. Jackson for editorial assistance. This work was funded by research grants from Joint Council Office (JCO) Development Programme, A-STAR core funding and A*STAR BMRC GRANT Singapore Consortium of Cohort Studies (SCCS; 05/1/21/19/425).

\section{CONFLICTS OF INTEREST}

No potential conflicts of interest were disclosed.

\section{REFERENCES}

1. Oosterlinck W, Sylvester R, Babjuk M, Kaasinen E, Bohle A, Palou-Redorta J, Roupret M: Should all patients receive an immediate chemotherapeutic drug instillation after resection of papillary bladder tumors? Eur Urol 2011, 59:374-376.

2. Cancer Genome Atlas Research N: Comprehensive molecular characterization of urothelial bladder carcinoma. Nature 2014, 507:315-322.

3. Reinert T, Modin C, Castano FM, Lamy P, Wojdacz TK, Hansen LL, Wiuf C, Borre M, Dyrskjot L, Orntoft TF: Comprehensive genome methylation analysis in bladder cancer: identification and validation of novel methylated genes and application of these as urinary tumor markers. Clin Cancer Res 2011, 17:5582-5592.

4. Allory Y, Beukers W, Sagrera A, Flandez M, Marques M, Marquez M, van der Keur KA, Dyrskjot L, Lurkin I, Vermeij $\mathrm{M}$ et al: Telomerase reverse transcriptase promoter mutations in bladder cancer: high frequency across stages, detection in urine, and lack of association with outcome. Eur Urol 2014, 65:360-366.

5. Miah S, Dudziec E, Drayton RM, Zlotta AR, Morgan SL, Rosario DJ, Hamdy FC, Catto JW: An evaluation of urinary microRNA reveals a high sensitivity for bladder cancer. $\mathrm{Br}$ J Cancer 2012, 107:123-128.

6. Hwang EC, Choi HS, Jung SI, Kwon DD, Park K, Ryu SB: Use of the NMP22 BladderChek test in the diagnosis and follow-up of urothelial cancer: a cross-sectional study. Urology 2011, 77:154-159.

7. Gutierrez Banos JL, del Henar Rebollo Rodrigo M, Antolin Juarez FM, Garcia BM: Usefulness of the BTA STAT Test for the diagnosis of bladder cancer. Urology 2001, 57:685689. 
8. Tilki D, Burger M, Dalbagni G, Grossman HB, Hakenberg OW, Palou J, Reich O, Roupret M, Shariat SF, Zlotta AR: Urine markers for detection and surveillance of non-muscleinvasive bladder cancer. Eur Urol 2011, 60:484-492.

9. Rosser CJ, Chang M, Dai Y, Ross S, Mengual L, Alcaraz A, Goodison S: Urinary Protein Biomarker Panel for the Detection of Recurrent Bladder Cancer. Cancer Epidemiol Biomarkers Prev 2014.

10. Rosser CJ, Ross S, Chang M, Dai Y, Mengual L, Zhang G, Kim J, Urquidi V, Alcaraz A, Goodison S: Multiplex protein signature for the detection of bladder cancer in voided urine samples. J Urol 2013, 190:2257-2262.

11. Swa HL, Shaik AA, Lim LH, Gunaratne J: Mass spectrometry-based quantitative proteomics and integrative network analysis accentuates modulating roles of Annexin-1 in mammary tumorigenesis. Proteomics 2014.

12. Cox J, Mann M: MaxQuant enables high peptide identification rates, individualized p.p.b.-range mass accuracies and proteome-wide protein quantification. Nat Biotechnol 2008, 26:1367-1372.

13. Sharma S, Zippe CD, Pandrangi L, Nelson D, Agarwal A: Exclusion criteria enhance the specificity and positive predictive value of NMP22 and BTA stat. J Urol 1999, 162:53-57.

14. Dieplinger $\mathrm{H}$, Ankerst DP, Burges A, Lenhard $\mathrm{M}$, Lingenhel A, Fineder L, Buchner H, Stieber P: Afamin and apolipoprotein A-IV: novel protein markers for ovarian cancer. Cancer Epidemiol Biomarkers Prev 2009, 18:11271133.

15. Liu W, Liu B, Cai Q, Li J, Chen X, Zhu Z: Proteomic identification of serum biomarkers for gastric cancer using multi-dimensional liquid chromatography and 2D differential gel electrophoresis. Clin Chim Acta 2012, 413:1098-1106.

16. Kollerits B, Krane V, Drechsler C, Lamina C, Marz W, Ritz E, Wanner C, Kronenberg F: Apolipoprotein A-IV concentrations and clinical outcomes in haemodialysis patients with type 2 diabetes mellitus--a post hoc analysis of the 4D Study. J Intern Med 2012, 272:592-600.

17. Chen YT, Chen CL, Chen HW, Chung T, Wu CC, Chen CD, Hsu CW, Chen MC, Tsui KH, Chang PL et al: Discovery of novel bladder cancer biomarkers by comparative urine proteomics using iTRAQ technology. J Proteome Res 2010, 9:5803-5815.

18. Jia T, Liu YE, Liu J, Shi YE: Stimulation of breast cancer invasion and metastasis by synuclein gamma. Cancer Res 1999, 59:742-747.

19. Iwaki H, Kageyama S, Isono T, Wakabayashi Y, Okada Y, Yoshimura K, Terai A, Arai Y, Iwamura H, Kawakita $M$ et al: Diagnostic potential in bladder cancer of a panel of tumor markers (calreticulin, gamma -synuclein, and catechol-o-methyltransferase) identified by proteomic analysis. Cancer Sci 2004, 95:955-961.

20. Rodrigues RG, Panizo-Santos A, Cashel JA, Krutzsch HC,
Merino MJ, Roberts DD: Semenogelins are ectopically expressed in small cell lung carcinoma. Clin Cancer Res 2001, 7:854-860.

21. Canacci AM, Izumi K, Zheng Y, Gordetsky J, Yao JL, Miyamoto H: Expression of semenogelins I and II and its prognostic significance in human prostate cancer. Prostate 2011, 71:1108-1114.

22. Nagakubo D, Taira T, Kitaura H, Ikeda M, Tamai K, Iguchi-Ariga SM, Ariga H: DJ-1, a novel oncogene which transforms mouse NIH3T3 cells in cooperation with ras. Biochem Biophys Res Commun 1997, 231:509-513.

23. Kim RH, Peters M, Jang Y, Shi W, Pintilie M, Fletcher GC, DeLuca C, Liepa J, Zhou L, Snow B et al: DJ-1, a novel regulator of the tumor suppressor PTEN. Cancer Cell 2005, 7:263-273.

24. Shinbo Y, Taira T, Niki T, Iguchi-Ariga SM, Ariga H: DJ-1 restores p53 transcription activity inhibited by Topors/ p53BP3. Int J Oncol 2005, 26:641-648.

25. He XY, Liu BY, Yao WY, Zhao XJ, Zheng Z, Li JF, Yu BQ, Yuan YZ: Serum DJ-1 as a diagnostic marker and prognostic factor for pancreatic cancer. J Dig Dis 2011, 12:131-137.

26. Lee H, Choi SK, Ro JY: Overexpression of DJ-1 and HSP90alpha, and loss of PTEN associated with invasive urothelial carcinoma of urinary bladder: Possible prognostic markers. Oncol Lett 2012, 3:507-512.

27. Sarosdy MF: The use of the BTA Test in the detection of persistent or recurrent transitional-cell cancer of the bladder. World J Urol 1997, 15:103-106.

28. Atsu N, Ekici S, Oge OO, Ergen A, Hascelik G, Ozen H: False-positive results of the NMP22 test due to hematuria. J Urol 2002, 167:555-558. 